\title{
Fragmentation functions at Belle
}

\author{
Rolf Seuster*it \\ University of Hawaii, USA \\ E-mail: seusterephys.hawaii.edu
}

Two measurements of fragmentation functions at the Belle detector are presented. A data sample of about $105 \mathrm{fb}^{-1}$ was used to determine precisely the charm fragmentation functions of $c \rightarrow$ $D^{+}, D^{0}, D^{*+}, D^{* 0}, D_{s}^{+}$and $\Lambda_{c}^{+}$. A second analysis based on a data sample of $\mathrm{xx} \mathrm{fb}^{-1}$ was used to determine the so-called Collins fragmentation function, which connects the transverse component of the spin of a quark with a measureable azimuthal dependence of the hadrons produced around the momentum axis of the quark.

International Europhysics Conference on High Energy Physics

July 21st - 27th 2005

Lisboa, Portugal

* Speaker.

${ }^{\dagger}$ A footnote may follow. 


\section{Introduction}

In recent years, the Belle experiment has made important contribution to flavour physics, by measurements about e.g. CP violation in the $B$ sector.

Two measurements are presented, which are performed at the Belle detector but in fields other than the $B$ sector: The first being a measurement of the charm quark fragmentation functions, see also [1], the second being the first direct measurement of the Collins fragmentation function, see also [2]. Experimentally, it is important to measure heavy quark fragmentation functions as their shapes are different from the corresponding functions for light quarks; such a measurement is furthermore straightforward, as very often hadrons containing heavy quarks can easily be identified. Their distributions give valuable input to MC generators, which must be tuned or modified to reproduce closely the measured distributions. A direct measurement of the Collins fragmentation function would allow one to access the quark transversity distribution.

\section{Charm Quark fragmentation function}

This analysis uses $87.7 \mathrm{fb}^{-1}$ of $e^{+} e^{-}$annihilation data taken at the $\Upsilon(4 \mathrm{~S})$ resonance ("onresonance data"), and $15.0 \mathrm{fb}^{-1}$ taken $60 \mathrm{MeV}$ below the resonance ("continuum data"), recorded at the Belle detector at the KEKB accelerator [3]. A detailed description of the Belle detector can be found in [4].

To minimise possible kinematic biases due to tight selection criteria for identified particles, only loose cuts on the particle identification of the stable particles have been applied. In general, the identification for each track was based on one or more likelihood ratios, which combined the information from the time-of-flight and Čerenkov counters and the energy loss $\mathrm{dE} / \mathrm{dx}$ in the drift chamber. Pions and kaons were separated by a single likelihood ratio, protons were separated by two likelihood ratios. The efficiencies $\varepsilon$ and misidentification probabilities $f$ for tracks from signal candidates have been measured in data, in all cases except the proton, $\varepsilon>95 \%$ and $f \leq 26 \%$. For the slow pion from the $D^{*+} \rightarrow D^{0} \pi^{+}$decay, all quality and identification requirements were removed to increase the efficiency.

The reconstructed hadron decay chains used in this analysis are the following:

$$
\begin{aligned}
& D^{0} \rightarrow K^{-} \pi^{+}, D^{+} \rightarrow K^{-} \pi^{+} \pi^{+}, D_{s}^{+} \rightarrow \phi \pi^{+}\left(\phi \rightarrow K^{+} K^{-}\right), \Lambda_{c}^{+} \rightarrow p^{+} K^{-} \pi^{+}, \\
& D^{*+} \rightarrow D^{0} \pi^{+}\left(D^{0} \rightarrow K^{-} \pi^{+}\right), D^{*+} \rightarrow D^{+} \pi^{0}\left(D^{+} \rightarrow K^{-} \pi^{+} \pi^{+}\right) \text {and } \\
& D^{* 0} \rightarrow D^{0} \pi^{0}\left(D^{0} \rightarrow K^{-} \pi^{+}\right) .
\end{aligned}
$$

The inclusion of charge-conjugate modes is implied throughout. For all charmed ground state hadrons, candidates whose masses were within $50 \mathrm{MeV}$ of their respective nominal mass were considered, narrowing it to $15 \mathrm{MeV}$ for the excited states $D^{* 0}$ and $D^{*+}$.

Fig. 1 shows the efficiency-corrected $\mathrm{x}_{\mathrm{P}}$ distributions for the three particles $D^{0} \rightarrow K^{-} \pi^{+}, D_{s}^{+} \rightarrow \phi \pi^{+}$ and $D^{*+} \rightarrow D^{0} \pi^{+}$in $e^{+} e^{-}$annihilation events. For $\mathrm{x}_{\mathrm{P}}>0.5$, the differential $\mathrm{x}_{\mathrm{P}}$ distributions of the on-resonance sample and the continuum sample have been combined by a weighted average, where the inverse of the squared statistical uncertainty was used as the weight. 

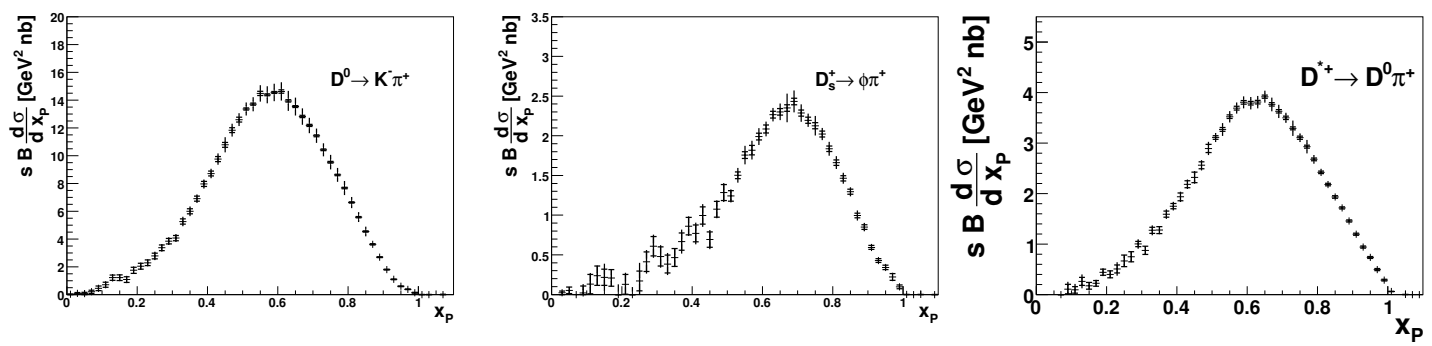

Figure 1: Efficiency corrected momentum distributions for $D^{0}, D_{s}^{+}$and $D^{*+}$ for $e^{+} e^{-}$-annihilation events. For $\mathrm{x}_{\mathrm{P}}>0.5$, the on-resonance and continuum data have been combined by a weighted average. The inner error bars show the statistical, the outer error bars the total uncertainty.

Various comparisons can be performed with the measured $\mathrm{x}_{\mathrm{P}}$ distributions, see [1] for more details. Via a reweighting technique, the data has been compared to several different fragmentation functions, their input parameters have been varied as well. The general trend is similar for all 5 fragmentation functions under consideration: The model by Bowler et al. showed the best agreement for almost all particles, the model by the Lund group, from which the Bowler model is derived, shows an almost similar well agreement. The other models considered here perform much worse, the model by Kartvelishvili $e$ al. and the model by Collins and Spiller have reduced $\chi^{2}$ about twice as large and the model by Peterson $e t$ al. shows the largest deviation from the data, see Table 1.

Table 1: The minimum of the chi-squared distribution, $\chi_{\min }^{2}$, for MC samples reweighted to represent the fragmentation functions shown, varying their respective parameters. The number of degrees of freedom (d.o.f.) is also shown for each case.

\begin{tabular}{cccccc} 
& $\mathrm{D}^{0}$ & $\mathrm{D}^{+}$ & $\mathrm{D}_{\mathrm{s}}^{+}$ & $\Lambda_{\mathrm{c}}^{+}$ & $\mathrm{D}^{*+}$ \\
\hline & $\chi_{\min }^{2} /$ d.o.f. & $\chi_{\min }^{2} /$ d.o.f. & $\chi_{\min }^{2} /$ d.o.f. & $\chi_{\min }^{2} /$ d.o.f. & $\chi_{\min }^{2} /$ d.o.f. \\
\hline Bowler & $1327.0 / 59$ & $188.4 / 60$ & $730.7 / 55$ & $269.1 / 60$ & $541.8 / 55$ \\
Lund & $1500.5 / 59$ & $527.1 / 60$ & $513.2 / 55$ & $266.6 / 60$ & $965.6 / 55$ \\
Collins and Spiller & $3032.1 / 58$ & $948.0 / 60$ & $1412.5 / 55$ & $2836.7 / 59$ & $1540.7 / 54$ \\
Kartvelishvili & $3210.4 / 59$ & $861.4 / 60$ & $735.3 / 55$ & $390.7 / 60$ & $1271.1 / 54$ \\
Peterson & $5070.2 / 59$ & $2229.6 / 60$ & $829.6 / 55$ & $1345.0 / 59$ & $3003.0 / 54$
\end{tabular}

\section{Collins Fragmentation Function}

The Collins fragmentation function describes the azimuthal distribution of hadrons produced from quarks with a transverse spin around the quark's momentum axis. In $e^{+} e^{-}$collisions this effect can be observed by measuring the azimuthal asymmetries between two hadrons produced in the fragmentation of a quark and an anti-quark in inclusive $e^{+} e^{-} \rightarrow \mathrm{h}_{1} \mathrm{~h}_{2} \mathrm{X}$ production. The thrust axis divides the event into two hemispheres. Out of each hemisphere, one hadron is selected, hadrons $\mathrm{h}_{1}$ and $\mathrm{h}_{2}$. 
Defining $\phi_{0}$ as the angle between the plane spanned by the momenta of $\left(\mathrm{h}_{1}, \mathrm{e}^{-}\right)$and the transverse momentum of $h_{2}$ w.r.t. the momentum of $h_{1}$. We study the $2 \phi_{0}$ distribution of all hadron pairs. In order to diminish detector effects that could introduce a non-zero azimuthal asymmetry we construct double ratios of $2 \phi_{0}$ distributions unlike sign and like sign pion pairs. The distribution can be parametrized as $\mathrm{p}_{1} \cos 2 \phi_{0}+\mathrm{p}_{2}$, where $\mathrm{p}_{1}$ depends on a convolution of the Collins fragmentation function over the transverse hadron momenta. A non-zero value of $\mathrm{p}_{1}$ thus reflects the asymmetry due to the Collins effect. The double ratio in a single bin of combined hadron pair momenta is shown in Fig. 2. Averaging the fitted $p_{1}$ values over the full range of momenta we find $\mathrm{p}_{1}=(3.26 \pm 0.56 \pm 0.55) \%$. This represents the first direct observation of the Collins effect.

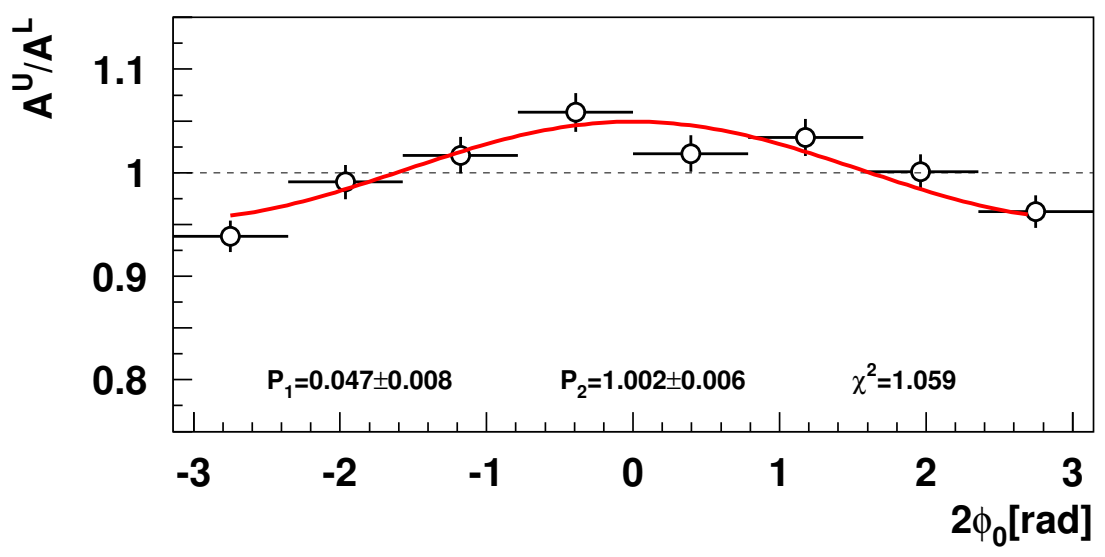

Figure 2: Asymmetry of unlike-sign over like-sign pion pairs.

\section{Summary}

A new determination of the charm fragmentation function at a CME close to the $\Upsilon(4 \mathrm{~S})$ resonance has been presented. The best agreement between data and $\mathrm{MC}$ has been found for the Bowler and the Lund models.

By measuring ratios of azimuthal asymmetries of unlike and like sign pion pairs we show a first direct evidence for the Collins effect in light quark fragmentation.

\section{References}

[1] Belle Coll., R. Seuster et al.: hep-ex/0506068

[2] Belle Coll., K. Abe et al.: hep-ex/0507063

[3] S. Kurokawa, E. Kikutani: Nucl. Inst. Meth. A499, 1 (2003);

and other papers included in this Volume.

[4] Belle Coll., A. Abashian et al.: Nucl. Inst. Meth. A479, 117 (2002). 